\title{
CALIDAD DE LA LEY, TÉCNICA LEGISLATIVA Y EFICIENCIA ADMINISTRATIVA*
}

\author{
Viviana Díaz Perilla**
}

Fecha de Recepción: 21 de septiembre de 2010

Fecha de Aceptación: 11 de enero de 2011

Artículo resultado de proyecto de investigación

\section{Resumen}

En el presente artículo se busca poner de presente la importancia que ha venido adquiriendo el tema relacionado con la calidad de la ley, tanto en otras latitudes como en nuestro medio; a partir del cual, se han elaborado diversas propuestas que buscan tecnificar los procesos de construcción normativa que le anteceden.

En éste se realiza un ejercicio de observación que permita establecer el grado de influencia que dichas propuestas han podido tener entre nosotros y, la manera cómo se armonizan con los mandatos constitucionales y principios rectores que dirigen las actuaciones de las diferentes autoridades estatales, entre las cuales se destaca la participación ciudadana.

\section{Palabras clave}

Ley, calidad, técnica, eficacia, administración pública, participación ciudadana, modernización del Estado.

\section{LAW QUALITY, LEGISLATIVE TECHNIQUE AND ADMINISTRATIVE EFFICIENCY}

\begin{abstract}
This article aims to highlight the growing importance of law quality in the local and international context, around which many proposals have been issued with the purpose of technify the normative construction processes that precedes them.

An exercise of observation has been in order to establish the degree of influence that the aforementioned proposals might have had among us, and the way in which such theoretical proposals are harmonized with the constitutional mandates and the ruling

* Este artículo hace parte de la investigación sobre participación ciudadana y administración pública dentro del proyecto "Sistemas de Garantías de los ciudadanos ante las administraciones públicas", iniciada en el mes de octubre de 2009 y actualmente en curso, en la que la autora es investigadora de la línea tendencias y perspectivas del derecho administrativo de la Facultad de Jurisprudencia de la Universidad del Rosario, institución que financia el proyecto.

** Abogada de la Universidad Externado de Colombia con Especialización en gerencia de Negocios Internacionales de la Universidad del Rosario, Becaria del Gobierno Francés y Graduada de la E.N.A Ėcole National d'Administration, promoción: Alliance des Civilisations, Mención: Magna Cum Laude en Administración Pública y D.S.U en Derecho Administrativo de la Universitè Panthèon Assas París II. Profesora de carrera académica y coordinadora académica de la Maestría en Derecho Administrativo de la Universidad del Rosario, investigadora de la línea de investigación "tendencias y perspectivas del derecho administrativo" de la Facultad de Jurisprudencia de la Universidad del Rosario. viviana.diaz@urosario.edu.co.
\end{abstract}


principles which lead the actions of the different state authorities. among which we can especially highlight citizen participation.

\title{
Key words
}

Law, quality, technique, efficiency, public administration, citizen participation, modernization of the State.

\section{QUALIDADE DA LEI, TÉCNICA LEGISLATIVA E EFICIÊNCIA ADMINISTRATIVA}

\begin{abstract}
Resumo
No presente artigo busca-se apresentar a importância que vem adquirindo o tema relacionado com a qualidade da lei, tanto em outras latitudes como em nosso meio, a partir do qual foram elaboradas diversas propostas que buscam tecnificar os processos de construção normativa que o antecedem.

Aqui é feito um exercício de observação que permite estabelecer o grau de influência que tais propostas podem ter tido entre nós, e a forma como se harmonizam com os mandatos constitucionais e princípios regentes que dirigem as ações das diferentes autoridades estatais, entre as quais se destaca a participação cidadã.
\end{abstract}

\section{Palavras-chave}

Lei, qualidade, técnica, eficácia, administração publica, participação cidadã, modernização do Estado.

\section{INTRODUCCIÓN}

Pese a los avances que se registran en las sociedades contemporáneas en los más diversos campos tales como la ciencia, la tecnología o las comunicaciones y a que ese conjunto de avances está puesto a disposición de todos aquellos quienes tienen bajo su responsabilidad la tarea de surtir los procesos de creación normativa, lo cierto es que hoy la calidad de los textos normativos sigue estando, como tradicionalmente lo ha estado, en entredicho.

Esta preocupación insistente por la calidad de las leyes y por incentivar el consecuente perfeccionamiento normativo está presente en todas las latitudes y se manifiesta de formas diversas, desde la crítica pura al papel de la ley, pasando por propuestas académicas que al integrar elementos científicos buscan tecnificar la elabora- ción y ejecución de las normas ${ }^{1}$ hasta posiciones mucho más radicales que plantean la necesidad de crear mecanismos jurídicos que permitan vincular la responsabilidad de los parlamentarios cuando la práctica indique que dieron cabida en el ordenamiento jurídico a una ley cuya calidad no satisface las expectativas sociales.

En el ámbito nacional el tema ha venido adquiriendo mayor importancia debido al aumento de la producción normativa, motivado, de una parte, por la necesidad de modernizar el Estado y de otra, por la necesidad de armonizar la legislación luego de la adopción de una nueva constitución en 1991.

A manera de ejemplo, resulta importante señalar la activa labor que desarrolla el "Grupo de Estudios de Técnica Legislativa” GRETEL en España. 
De esta forma, hemos visto incrementarse en el país las iniciativas legislativas que persiguen el aseguramiento de una mejor administración pública, de una adecuada impartición de justicia y de concretizar los principios establecidos por el nuevo texto constitucional.

De manera simplemente ilustrativa pueden mencionarse como ejemplos la expedición en 1998 de un nuevo estatuto de la administración pública, en 2004 la adopción de nuevas disposiciones en materia de carrera administrativa y de manera mucho más reciente la iniciativa de reformar el código contencioso administrativo que ha estado vigente desde hace más de 20 años y que se concretó a fines del año 2007.

Todas estas iniciativas se originaron con posterioridad a 1991 y por lo tanto en su concepción debieron ser tenidos en cuenta los nuevos principios constitucionales llamados a definir la forma en la que deben concebirse y desarrollarse el conjunto de actividades del Estado dentro de las que se encuentran los procesos de creación de las normas jurídicas.

Frente a este último punto, bástenos con imaginar, por ejemplo, la importancia que para el ejercicio de la función legislativa puede tener la concepción de participación ciudadana que se desarrolla en el nuevo texto constitucional en su doble carácter de principio fundamental $y$ de fin esencial del Estado que impone a sus autoridades la obligación de contribuir a la promoción de espacios de participación ciudadana en todos los ámbitos, dentro de los cuales se encuentra la posibilidad de incidir de manera importante en los procesos de construcción normativa.

Del mismo modo, se debe resaltar la importancia que para orientar la actividad de la administración, tuvo la consagración en el nuevo texto constitucional de los principios rectores para el ejercicio de la actividad administrativa y muy especialmente la de los principios de eficiencia y eficacia cuya consecución impone rediseñar tanto la composición organizacional como metodológica de la administración el ánimo de obtener el más alto nivel de rendimiento y productividad. (ZÁRATE PÉREZ: 2004: 66)

Estos principios pasan a ser un mandato constitucional de obligatorio cumplimiento para el conjunto de autoridades del Estado, por lo cual es bajo esa misma concepción que deben ser pensados los procesos de modernización del Estado y, por ende los procesos de construcción normativa que los acompañan, más aún cuando se verifica un alto índice de congestión a nivel jurisdiccional y un importante nivel de insatisfacción por parte de los administrados que pone en entredicho la capacidad de las leyes para responder adecuadamente a las necesidades ciudadanas. Es por ello que resulta pertinente hacer algunos comentarios alrededor del rol de los procesos de construcción normativa en procesos que, como el de modernización del Estado, buscan ofrecer alternativas innovadoras, así como establecer si en el proceso mismo de formación legislativa se han presentado transformaciones o si por el contrario, buscamos modernidad a través de trabajos legislativos pegados a la tradición; al "savoir faire la loi" dentro de una concepción clásica propia del Estado clásico y no de un Estado moderno contemporáneo, lo cual construirá el objeto del presente trabajo.

Habiéndose evidenciado dos variables: la necesidad de mejorar la calidad de la ley como requisito fundamental para alcanzar los objetivos del Estado y la sobreproducción normativa en Colombia, resulta interesante correlacionarlas para establecer si entre nosotros se han adoptado mecanismos de técnica legislativa en la concepción de las nuevas normas y si los principios constitucionales que dirigen la actividad del Estado han permeado de manera efectiva la actividad de creación normativa.

Para dar respuesta a las inquietudes planteadas, se acudió a la implementación de la metodología correlacional de investigación a partir de la cual se diseñó un plan de trabajo compuesto por tres etapas: en primer lugar se presentarán de 
manera muy general las transformaciones que ha experimentado el derecho desde la concepción que de él se tenía en el Estado moderno, para luego describir cómo esas transformaciones dieron lugar en la postmodernidad a una nueva disciplina que tendría como finalidad racionalizar los procesos normativos denominada legística (I), pasando luego a establecer la forma en la que las comisiones de reforma desarrollan su actividad (II) finalizando con una mirada a la experiencia colombiana y particularmente la más reciente relativa a la reforma al código contencioso administrativo (III).

\section{DE LOS FUNDAMENTOS CLÁSICOS DEL DERECHO A LA DINÁMICA CONTEMPORÁNEA}

La aparición del Estado moderno implicó modificaciones importantes para el derecho. Como resultado de su laicización, el Estado pasa a ser la única fuente de derecho y el poseedor del monopolio de la fuerza. (ELIAS: 1975) Sin embargo, si bien la supremacía del Estado era indiscutible, los ciudadanos entendían participar en el proceso de formación de la ley únicamente a través de sus representantes.

La función principal del derecho era enmarcar los comportamientos de los asociados en búsqueda de estabilidad y orden, percibidos como fundamentales para el progreso social. Dicha tarea debía ser cumplida a través del establecimiento de un conjunto sistematizado de normas de carácter general y estable. Es con el advenimiento del Estado proteccionista que el derecho es utilizado ya no sólo como instrumento de control social, sino como una herramienta que le permite al Estado dirigir sus esfuerzos hacia la consecución de objetivos relacionados con políticas sociales, de intervención en la actividad económica y, en general de medidas tendientes a restablecer el equilibrio social. (CHEVALLIER: 2002: 94)

Es a partir de la modificación que se fue produciendo en la concepción frente a las funciones a las cuales estaban llamadas a responder las normas jurídicas y, específicamente en lo relacionado con el logro de objetivos precisos que van más allá del establecimiento y mantenimiento del orden, que se produjo una cierta flexibilización frente a la técnica jurídica utilizada; al permitirse, por ejemplo, acudir a mecanismos extrajurídicos que facilitaran responder al imperativo de eficacia que se había instalado. (CHEVALLIER: 2002: 94)

De este modo, se pasó a un concepto de derecho regulador que puso de manifiesto el replanteamiento de los beneficios que la ley parecía ofrecer pasándose a preferir el reglamento, de esta forma se incrementó la producción normativa pegada al detalle y a los tecnicismos de manera acelerada $y$ desordenada; lo cual condujo en un momento posterior, al desarrollo de un movimiento de desreglamentación en búsqueda de la simplificación perdida, al que sucedió una nueva visión frente a la forma en la que debía abordarse esa búsqueda de la eficacia que pone de manifiesto una vez mas la vieja aspiración de contar con un derecho simple y accesible.

Al respecto vale decir que se busca abordar el derecho con un enfoque mucho más pragmático, creativo, flexible, adaptable, plural, producto de la asociación de los destinatarios al proceso de elaboración de las normas y en permanente proceso de aprendizaje, en suma un derecho producto de una mayor reflexión.

De acuerdo con lo expuesto, es posible concluir que las normas jurídicas pueden ser tanto instrumento de desarrollo como freno a los objetivos trazados, no puede por lo tanto pensarse en un proyecto de modernización cuyas normas sean demasiado etéreas ni en un proyecto edificado a partir de normas demasiado detalladas y en número tan excesivo que su aplicación resulte desgastante y tortuosa.

Los procesos de construcción normativa están llamados a encontrar el justo medio, tarea por demás, nada sencilla. Ese justo medio podría encontrar como fuente los elementos enunciados que responden a dos de las preocupaciones 
fundamentales del derecho hoy: la eficiencia y la practicidad. En esos términos, la creatividad, la adaptabilidad, la autorreflexión y la negociación con los gobernados deberán estar integradas en el desarrollo de la tarea legislativa. Desde luego, ello contribuirá a hacer de los procesos una labor mucho más larga y dispendiosa, sin embargo, lo que sería de esperar es que su resultado justificara en gran medida el esfuerzo.

Una labor tan dispendiosa como esta necesita, para ser exitosa, estar acompañada una metodología adaptada y de técnicas que permitan la adecuada interacción de los que en ella participen, por lo cual hoy es posible hablar de una tecnificación de los procesos de construcción normativa.

\subsection{La Tecnificación de los Procesos de Construcción Normativa}

Hacia los años setenta ${ }^{2}$ hizo su aparición en el mundo jurídico una disciplina que describe la preocupación por tecnificar la producción normativa que se denominó legística.

La legística nació como resultado de la preocupación alrededor de la calidad de las normas jurídicas y tiene como objetivo establecer las mejores modalidades posibles para la concepción, elaboración, redacción, edición y aplicación de las normas de derecho.

El concepto de esta disciplina está formado por dos enfoques: el de la legística material y el de la legística formal. La primera acepción se refiere a los principios y conocimientos que permiten reforzar la eficacia de la legislación; en otras palabras, la legística material trata sobre el contenido de la materia que debe ser reglamentada y determina la forma mediante la cual un problema está reglamentado o debe ser reglamentado dejando a la segunda la preocupación sobre la forma de mejorar la comunicación legislativa durante la

2 El término "legística" apareció por primera vez en 1973 en la obra titulada Gesetzgebungslehre del Profesor Alemán Peter Noll. etapa en la que un proyecto normativo pasa a ser un texto legal ${ }^{3}$.

De acuerdo con las palabras del profesor Jacques Chevallier, quien se ha ocupado de estudiar este tema, la legística "busca estudiar la actividad de producción normativa y definir las técnicas que mejor se adapten a la gestión de dicha producción: se trata no solamente de favorecer un mejor conocimiento de las normas existentes utilizando los recursos ofrecidos por Internet, sino también de asegurar la actualización de los dispositivos jurídicos así como de mejorar la redacción y la formulación de los textos". (CHEVALLIER: 2011: 132)

La tecnificación de los procesos pasa entonces por la utilización de modernas herramientas tales como las nuevas tecnologías de información y de comunicación -TICS- que vienen a fortalecer los mecanismos tradicionales de participación de los ciudadanos en la elaboración de las normas, tales como las encuestas o las conferencias en las que interactúan expertos, ciudadanos y en general, los portavoces de los diferentes intereses sociales.

Dichos mecanismos facilitan convocar no solamente a los participantes, sino promover los procesos consultivos y de discusión en escenarios igualmente novedosos tales como foros virtuales; así como procesar de manera más rápida y eficiente la información obtenida.

Así las cosas, si el objetivo fundamental es el mejoramiento de las condiciones de producción del derecho no sólo es menester contar con una metodología adaptada, que cumpla tanto material como formalmente con los objetivos sino que además ésta debe integrarse con una concepción reflexiva de base que le sirva de sustento.

3 Definición dada por el antiguo vicepresidente del Consejo de Estado Francés Renaud Denoix de SaintMarc y por el antiguo secretario general de Gobierno Jean- Marc Sauve en Rapport public du Conseil d'Etat 2003, Considérations générales: Perspectives pour la fonction publique (EDCE $\left.n^{\circ} 54\right), 2003$. 
En grandes rasgos, las características principales de la regulación jurídica reflexiva pueden resumirse en dos. En primer lugar, ésta deberá buscar la organización de la democratización de la democracia, legitimando la intervención de espacios subpolíticos en los procesos normativos $y$, de otra parte prever desde el comienzo las condiciones de su evolución frente a las dificultades constatadas en la práctica. (PIRET, J.)

Esta famosa expresión de Ulrich Beck "democratizar la democracia" (BECK. \& WILLMS. 2004) que puede sonar tautológica, cobra sentido si se explica a través de la ampliación de los espacios de participación de la sociedad civil, de las organizaciones no gubernamentales $y$ en general de los nuevos actores que influyen hoy, directa o indirectamente, en el devenir de lo público. De tal suerte que "el derecho vuelva legítimo y transparente aquello que aún hoy se limita a un lobby ampliamente confidencial o conducido por grupos de presión más o menos discretos" (HOECKE, V: 2002).

Es a esa misma lógica a la que deberían responder los procesos de modernización cuya dinámica sea reflexiva pues, de acuerdo con el autor antes citado, dichos procesos son capaces de cuestionarse a sí mismos. Esa capacidad de auto cuestionamiento no debe ser interpretada como un análisis que sólo convoque a los responsables de su diseño y puesta en marcha, sino que debe complementarse con una mirada desde fuera, desde diversas esferas que hagan de ese ejercicio una verdadera fuente de funcionalidad de las medidas adoptadas a posteriori.

De modo tal que lo que debe haber de fondo es una modificación del pensamiento que permita movilizar a los actores contemporáneos que forman parte de los procesos normativos hacia formas alternativas de comportamiento que posibiliten privilegiar la reflexión individual sólo como punto de partida hacia una reflexión colectiva, (MAESSCHALCK: 2008) basada en las fortalezas argumentativas de los individuos; de este modo, se consolida una verdadera interacción que da mayor legitimidad al proceso.
Sólo así será posible pasar del "poder hacer" característico de los hacedores del derecho clásico al "saber hacer" y el "poder cambiar aquello que está hecho" que debe caracterizar a los constructores del derecho contemporáneo.

Sin embargo, dicha legitimidad no surge exclusivamente de la colectivización de la tarea reflexiva desde lo individual, sino que además requiere, de acuerdo con lo expuesto, que se concierten mecanismos de autoevaluación y por tanto el desarrollo de la capacidad interna de efectuar las reformas que se requieran; de modo que el proceso de construcción se enriquezca con la experiencia y resultados no sólo de otros procesos similares sino del que se encuentra en curso.

Esta referida tecnificación, se nutre además de métodos adaptados y de sostenidas reflexiones, $\mathrm{de}$ instrumentos que pueden ser tomados de experiencias exitosas no necesariamente legislativas; dando paso a lo que puede denominarse la gerencia normativa.

\section{2 La Gerencia Normativa}

En este punto, no cabe duda que al hablar de proceso de creación normativa se piense de inmediato en un asunto no sólo complejo sino dinámico. Dicha dinamicidad, tal como ha sido expuesto, está directamente relacionada con las transformaciones mismas que presenta el Estado fruto, entre otras, de la evolución social y de los movimientos económicos que a su vez permiten proveer la labor normativa, incluso con herramientas que hasta el momento resultaban ajenas a la tarea legislativa y que parecerían más propias de otras actividades de índole privado.

Piénsese por ejemplo, en los instrumentos propios del Management que paulatinamente impregnaron la actividad de la administración, a tal punto que hoy no se concibe la labor de direccionamiento de lo público sin una óptica gerencial cuyos principios fundamentales están dirigidos a satisfacer justamente la necesidad de lograr objetivos de la manera más eficaz posible. 
Este por supuesto, ha sido un fenómeno global que no tardó en influir en la administración pública colombiana a tal punto que sus premisas básicas están hoy presentes en todos los niveles de la actividad administrativa, desde el texto constitucional hasta los planes nacionales de desarrollo.

Por ello, pese a que del modelo gerencial se ha dado paso a una nueva gobernanza no podría afirmarse válidamente, por lo menos en el caso colombiano, que ha operado una sustitución del modelo; sino que por el contrario, podría hablarse de una sinergia por cuanto se conservan instrumentos tales como la planeación, la evaluación y el control de resultados propios del modelo gerencial a los que se ha sumado una preocupación insistente por incrementar la participación ciudadana en su consideración más amplia, esto es, abriendo paso a que nuevos actores formen parte activa de lo que antes era de resorte exclusivo y en algunos casos excluyente de las autoridades administrativas, nota característica de la gobernanza.

De esta forma, no resulta descabellado pensar en la adopción, en el más literal de los sentidos, de mecanismostalescomoelbenchmarking, lacalidad total o el empoderamiento (empowerment), entre muchos otros. El benchmarking, por ejemplo, se define como un proceso sistemático, continuo de investigación y aprendizaje para evaluar los productos, servicios y procesos de trabajo de las organizaciones que son reconocidas como representantes de las mejores prácticas, con el propósito de realizar mejoras organizacionales. (KONNTZ. \& WEIHRICH: 1998)

Porsuparte, lacalidad total implicalaparticipación continua de todos los trabajadores de una organización en la mejoría del desarrollo, diseño, fabricación y mantenimiento de los productos y servicios que ofrece una organización. De igual manera, esta participación debe verse reflejada en las actividades que se realizan dentro de la misma. (WALTON: 1992)

Finalmente, el empoderamiento significa crear un ambiente en el cual los empleados de todos los niveles sientan que tienen una influencia real sobre los estándares de calidad, servicio y eficiencia del negocio dentro de sus áreas de responsabilidad. Esto genera por una parte, sentido de compromiso y autocontrol y, por otra parte el hecho de que los empleados, administrativos o equipos de trabajo posean el poder para la toma de decisiones en sus respectivos ámbitos, implica aceptación de responsabilidad por sus acciones y tareas. (KONNTZ. \& WEIHRICH: 1998)

Frente a lo anteriormente expuesto, interesa detenerse en los puntos de convergencia entre un discurso eminentemente empresarial y otro que aunque ha dejado de ser exclusivamente estatal mantiene como es natural, una línea eminentemente pública.

De esta observación resultan varios elementos comunes, en primer lugar la necesidad de implementar herramientas de evaluación que vayan más allá de la propia actividad y en segundo lugar, una mayor participación en los procesos de los diferentes estamentos; participación que no se limita a lo meramente operativo, sino que abarca el ámbito de lo decisional generándose procesos de autocontrol o de autorreflexión y por ende, asunción de responsabilidades por los resultados de la actividad realizada.

En este punto, es importante aclarar que este ejercicio comparativo no pretende asimilar el derecho a un producto o a un servicio que se obtiene como resultado de un proceso normativo industrializado deshumanizándolo o, dejando de lado los valores que le son propios tales como la protección del interés general o la búsqueda de la justicia, sino que simplemente responde a un interés por ver cómo la búsqueda de la eficiencia en el sector público y en el sector privado ha recorrido las mismas etapas, ha establecido los mismos parámetros $y$, ha obtenido resultados similares que invitan a continuar avanzando hacia una mayor tecnificación para la obtención de un mejor desempeño en sus respectivas tareas.

Valga la pena mencionar a título de ejemplo, la reciente expedición en Colombia del Decreto 
1345 del 23 de abril de 2010, mediante el cual se adoptaron directrices de técnica normativa para la elaboración de decretos y resoluciones. Esta es una clara muestra de la preocupación que hay actualmente en el país por incrementar la eficacia de las normas a través de la adopción de técnicas normativas, en aspectos tales como la estructura, presentación uniforme de las mismas, su lenguaje y redacción; lo cual se espera redunde en una mejor comprensión y aplicación de la normatividad, que facilite a los ciudadanos conocer sus derechos y obligaciones, y que, en palabras del Decreto, las autoridades administrativas tengan un marco idóneo, claro, coherente y funcional a partir del cual puedan desempeñar correctamente el ejercicio de sus competencias.

También es importante señalar, que con la expedición de este Decreto se ha evolucionado pues constituye un intento por pasar de "determinar las exigencias formales para la elaboración de Decretos y resoluciones", finalidad del Decreto que le antecedía a "establecer las directrices de técnica normativa" dirigidas a mejorar las normas que le corresponde expedir al Gobierno y a las entidades pertenecientes a la rama ejecutiva del poder público.

Este decreto, tanto en su articulado como en el manual de técnica legislativa que lo acompaña, contiene elementos muy importantes desde el punto de vista gerencial tales como la necesidad de realizar un análisis previo a la adopción de normas de la viabilidad jurídica, fáctica y de conveniencia de las mismas; la planeación en su elaboración; la coordinación entre las diferentes entidades interrelacionadas y, el estudio previo del impacto social y económico, especialmente respecto de los grupos o personas que son objeto de especial protección constitucional.

Ese es de manera general el contexto actual en lo atinente a la labor realizada al interior de la rama ejecutiva en el país; sin embargo, podríamos válidamente preguntarnos acerca de la posibilidad de que la influencia de esos mecanismos administrativos haya tocado las esferas de las competencias propias de la rama legislativa, o en otros términos: ¿podría pensarse en la labor normativa como en una labor gerencial?

Podría pensarse que una respuesta en sentido positivo a un planteamiento como el anteriormente expuesto no significaría la desnaturalización de los procesos de construcción normativa en cuanto al sentido conceptual y filosófico que les es propio, sino que podría representar un medio adicional que facilitara el avance hacia la regulación jurídica reflexiva.

De ello también parece dar fe el Congreso de la República colombiano ya que en vigencia de la actual carta política colombiana expidió la ley 1114 de 2007, cuyo objeto era la transformación integral y progresiva del Congreso de la República en una institución legislativa moderna, altamente técnica y capaz de responder de manera eficaz y eficiente a las exigencias de la democracia. Como parte de los mecanismos adoptados para el logro de dichos objetivos, se adoptó el Sistema de Información Parlamentaria y se crearon tres comisiones especializadas: la "Comisión Especial de Modernización del Congreso", la "Unidad Coordinadora de Asistencia Técnica Legislativa del Congreso de la República" y la "Unidad Coordinadora de Atención Ciudadana del Congreso de la República".

Este es entonces, el marco general de la integración de elementos innovadores que buscan facilitar la tarea normativa en el plano teórico, sin embargo, su concreción en el mundo de lo real no es tan sencilla, es por ello que resulta interesante ver lo que ocurre al interior de las comisiones de trabajo encargadas de redactar proyectos de ley.

\section{La Dinámica de las Comisiones de Reforma}

Las comisiones de reforma del derecho se conforman normalmente como resultado de la iniciativa gubernamental con miras a que un comité de expertos concrete sus recomen- 
daciones en un proyecto de ley, proyecto que posteriormente deberá surtir los procedimientos constitucionalmente previstos para obtener legítimamente la aprobación del cuerpo legislativo y su posterior sanción presidencial.

De este modo, las comisiones de reforma son convocadas e instituidas esencialmente al interior del poder político sin que por ello sus recomendaciones resulten obligatorias o vinculantes, sino que se espera, sirvan como punto de partida a la discusión legislativa cimentada sobre bases sólidas desde el punto de vista de técnica legislativa. En esto, las comisiones deben ser expertas así como en el tema en torno al cual debe girar la actividad de creación normativa, puesto que si bien el Congreso, o de manera más general, los parlamentos pueden estar llenos de buenas intenciones no necesariamente son expertos en el cúmulo de materias sobre las cuales deben legislar.

De conformidad con la teoría de los sistemas de Luhmann, (BOURDIEU \& TEUBNER: 2000: 123) que busca dar respuesta al problema que se presenta en la sociedad moderna acerca de la dificultad que para el logro de la integración normativa constituyen los conflictos inherentes $y$ potencialmente desintegradores entre las distintas racionalidades de los subsistemas político, económico y cultural; puede decirse que el sistema jurídico moderno integra esencialmente dos formas de operación: las que surgen de la auto observación del derecho y las que provienen de su auto descripción. (DUBÈ, R : 25) Dados entonces esos conflictos, se plantea la necesidad de propiciar mecanismos de integración que faciliten el trabajo por ejemplo, de las comisiones de reforma las cuales, de acuerdo con lo anteriormente planteado vienen operando o desde la auto observación del derecho o bien desde su auto descripción.

De acuerdo con quienes han estudiado en profundidad el tema, las operaciones de producción normativa primitivas son producto de una actividad realizada a partir de la auto observación dado su carácter cerrado y pegado a la forma y a los procedimientos caracterizado por limitar los espacios, destinado a aprender de las experiencias pasadas por lo cual es frecuente que se rechacen de plano los cambios sugeridos.

La auto observación invita a la construcción a partir de lo ya construido sin permitirse opciones innovadoras, creativas, que provengan de fuentes de información y/o de aprendizaje diferentes a las jurídicas, cerrando la puerta a la crítica que provenga de sectores distintos a los jurídicos; por lo cual, por ejemplo, la opinión de sectores académicos o profesionales de otras áreas no es tenida en cuenta, de este modo se corre el riesgo de sacrificar el contenido o la finalidad en procura de respetar las tradiciones, los procedimientos y las formas.

De otra parte, la auto descripción permite recurrir a fórmulas distintas a las jurídicas permitiéndose por ejemplo, que se tomen en consideración críticas, observaciones o sugerencias de orígenes diverso; se fomenta incluso espacios que las propicien pues se parte del supuesto de que ellas constituyen una fuente invaluable de aprendizaje y por lo tanto, un importante punto de partida para el fomento de procesos creativos.

Del mismo modo, la auto descripción implica la apertura hacia procesos reflexivos en los que confluyen cuestionamientos sobre sí mismos, mecanismos de auto evaluación y por ende, mecanismos de auto corrección, que si bien no garantizan en sí mismos un proceso de creación normativa plenamente eficaz o innovador, si permite mayor flexibilidad y adaptación del derecho.

De lo anteriormente expuesto, puede concluirse que las comisiones de reforma cuyo interés esté dirigido hacia la formulación de propuestas normativas innovadoras deberían contemplar una metodología de trabajo flexible y abierto a procesos de auto aprendizaje y de autocorrección.

Varios son los beneficios que se desprenden del hecho de que sea una Comisión de reforma la que se encargue de elaborar un proyecto de ley y no necesariamente el cuerpo parlamentario, 
dentro de los cuales se encuentran: la experticia técnica que convoca a los elegidos para conformar esas comisiones como la neutralidad política de los mismos que les permite un campo de acción más amplio y menos condicionado, la temporalidad que lleva implícita la posibilidad de tener el tiempo necesario para realizar un proceso reflexivo y la posibilidad de contar con una mayor libertad de pensamiento ${ }^{4}$.

La demanda de producción normativa es tan dinámica como la sociedad misma, la evolución tecnológica conlleva el surgimiento nuevas materias que deben ser revisadas desde el punto de vista normativo, lo cual implica un esfuerzo para el que no siempre la estructura interna y logística de los parlamentos está preparada.

Esta es la razón por la cual, de encontrarse frente a la necesidad de intervenir en asuntos álgidos, complicados o de gran trascendencia nacional, los parlamentos no pueden suspender el resto de sus actividades y preferirlos frente a otros igualmente importantes aunque menos complejos para dedicarse de forma exclusiva a ese proyecto de tanta trascendencia dejando de lado el cumplimiento de sus funciones so pena de causar perjuicios importantes para los asociados. Como resultado, se hace necesario trasladar una parte del proceso a un grupo de expertos que pueda dedicar el tiempo necesario para desarrollar un proceso de profunda reflexión que no hubiera podido ser realizado en otras condiciones y que resulta muy beneficioso frente a la calidad del producto final.

Así, por ejemplo en el caso de la reforma del código contencioso administrativo, la comisión contó con un término que aunque inicialmente se estableció de un año, se extendió a otro adicional; tiempo en el que fue posible convocar la opinión de expertos nacionales e internacionales sobre los aspectos más importantes de la reforma así como tener en cuenta iniciativas provenientes de otros ámbitos como los académicos o las opiniones $e$ intervenciones de los integrantes de la rama

4 Contribuciones conceptuales aportadas por Michael Foucault y Niklas Luhmann. judicial y de los ciudadanos, lo cual hubiese sido desde el punto de vista temporal y logístico prácticamente imposible para el congreso.

Del mismo modo, la especialidad que revisten ciertos asuntos impone la necesidad de que la producción normativa sobre los mismos sea atendida por expertos, quienes podrán generar debates mucho más fructíferos y fértiles que permitan la adopción de mejores propuestas normativas que brindarán un apoyo invaluable a la labor posterior del congreso.

Finalmente, la mayor libertad de pensamiento es un reflejo de la autonomía con la que cuentan las comisiones de las que se espera no solamente neutralidad desde el punto de vista de las influencias políticas, sino también neutralidad frente a propuestas legislativas pasadas, directrices definidas por partidos políticos frente al manejo de ciertos asuntos decisiones judiciales tomadas con anterioridad al inicio de sus actividades sobre los temas frente a los cuales debe pronunciarse.

Dado que en la actualidad se encuentra en trámite legislativo el proyecto de reforma al Código Contencioso Administrativo colombiano, el cual es producto del trabajo que durante aproximadamente dos años realizó la Comisión para la Reforma a la Jurisdicción Contencioso Administrativa creada por iniciativa gubernamental mediante el Decreto 4820 del 14 de diciembre de 2007; vale la pena plantear algunos puntos de reflexión acerca de un proceso de construcción normativa que como este, constituye una etapa obligada hacia la modernización de la jurisdicción contenciosa cuyo marco de referencia se encuentra en un proyecto mucho más ambicioso de modernización del Estado.

\section{EL PROCESO DE CONSTRUCCIÓN NORMATIVA PREVIO A LA REFORMA DEL CÓDIGO CONTENCIOSO ADMINISTRATIVO COLOMBIANO}

La jurisdicción contenciosa en Colombia fue constitucionalmente concebida desde la Carta 
de 1886, pero fue sólo hasta 1913 y con la finalidad de dar cumplimiento a lo establecido por el Acto Legislativo número 3 de 1910 que se expidió una ley en la que se consolidó y estructuró la jurisdicción, ley que pasó a convertirse en el primer código contencioso del país.

La evolución normativa en materia contenciosa administrativa en Colombia es relativamente corta si se tiene en cuenta que en un lapso de 97 años se han expedido tres códigos, los cuales si bien fueron modificados en varias oportunidades conocieron amplios periodos de estabilidad. El primer código ya mencionado, data de 1913, el segundo fue expedido en 1941 y el último cuya reforma fue recientemente promovida está vigente desde el año 1984.

Frente al tema que nos ocupa, esto es la reflexión acerca de los procesos de construcción normativa, cabe mencionar cómo en la expedición del primer código hubo una activa participación de los que para entonces, y de acuerdo con el criterio del gobierno fueron calificados como eminentes juristas, sin que se hubiese contemplado la posibilidad de ampliar el ámbito de participación a otros frentes de opinión.

En cuanto al segundo código, éste fue redactado por el mismo Consejo de Estado con la participación de algunos profesores universitarios, pero fue sólo hasta la década de los ochenta en que por primera vez se conformó una Comisión Asesora del Gobierno ${ }^{5}$, para reformar el código, presidida por el Ministro de Justicia o su delegado e integrada además por dos Senadores y dos Representantes, designados por las Mesas Directivas de las respectivas Comisiones Primeras; dos Magistrados del Consejo de Estado, uno de la Sala Contencioso-Administrativa y uno de la Sala de Consulta y Servicio Civil, elegidos por las Mesas Directivas correspondientes; así como dos profesores de Derecho administrativo, nombrados por la Academia de Jurisprudencia.

5 La Comisión Asesora del Gobierno para la reforma al Código Contencioso Administrativo fue creada por la ley 58 de 1982, artículo 12 .
Este es entonces el antecedente más cercano de un trabajo de reforma normativa para la jurisdicción contenciosa fruto del esfuerzo mancomunado de una Comisión de expertos convocada por el gobierno nacional. En esa oportunidad, se partió, como es natural, del criterio de la experticia en derechoadministrativo y materias afines para la conformación del grupo de trabajo; sin embargo, el texto legal que dio origen a su creación limitó al conocimiento exclusivo de sus miembros la labor de reforma.

Debido a que el primero de agosto de 2006 entraron en operación 257 juzgados administrativos $^{6}$, se presentó un incremento en la carga laboral dentro de la jurisdicción; puesto que, los nuevos despachos judiciales iniciaron labores debiendo conocer tanto los procesos que recibieron como herencia de los tribunales administrativos como los nuevos para los que, de conformidad con la ley eran competentes.

Esta carga laboral y el consecuente represamiento de los procesos hicieron necesaria la implementación de medidas de descongestión judicial tales como la vinculación de personal nuevo y con carácter transitorio para superar la crisis que los estudios estadísticos mostraban como inminente. Sin embargo, en el ambiente se asumió la idea de que medidas como esa iban a resultar insuficientes por lo cual se hacía necesario avanzar de manera mucho más radical hacia el fortalecimiento de la jurisdicción a través de la reducción de los procedimientos judiciales y la posible implementación de la oralidad; lo que constituyó, además de la necesidad de armonizar la normatividad en lo contenciosos con el nuevo texto constitucional vigente, el motivo principal para consolidar la reforma a la jurisdicción contencioso administrativa.

Es dentro de este marco que se expidió el Decreto 4820 de 2007 en cuyo articulado pueden verse algunas diferencias con respecto a la experiencia inmediatamente anterior. Partiendo de lo

$6 \quad$ Estos 257 juzgados están distribuidos actualmente en 26 distritos y 38 circuitos. 
simple, se observa en una primera instancia que la iniciativa para reformar el código en 1982 fue de origen legal otorgándole al Presidente de la República facultades extraordinarias para cumplir con dicha tarea y creando una Comisión Asesora del gobierno en ese mismo sentido. Al mismo tiempo, se introdujeron algunas reformas al código vigente que se consideraban de vital importancia para el adecuado desarrollo de las actuaciones administrativas, dentro de las que se destacan en razón a su importancia, la inclusión por primera vez de los principios de economía, celeridad, eficacia e imparcialidad como los llamados a orientar el ejercicio de la actividad de la administración, los cuales fueron posteriormente complementados y precisados en el código expedido en 1984.

De acuerdo al contenido de la mencionada ley, puede inferirse que para ese momento el interés del Congreso estaba más dirigido a precisar los grandes temas que debían ser objeto de reforma que a pronunciarse en detalle sobre las funciones, modalidades o criterios; aspectos importantes para el desarrollo del objeto para el que había sido creada la comisión asesora del gobierno. Ello resulta lógico porque era el propio texto constitucional el que imponía que en el contenido de la ley, mediante la cual se concedían facultades extraordinarias al Presidente, éstas debían estar claramente precisadas.

Por el contrario, la reforma a la jurisdicción contenciosa propuesta en el 2007 tiene como origen una iniciativa gubernamental que se concretó en un Decreto, a través del cual se observa un claro interés por definir con mayor precisión tanto las funciones como la forma en la que se debe desarrollar el trabajo de la Comisión fruto de un articulado que sin ser extenso permite fijar algunos lineamientos generales. De esta forma, como únicas precisiones alrededor del contenido mismo de la reforma se incluyeron la necesidad de determinar la viabilidad de implementar la oralidad en la jurisdicción y la reducción de los diferentes procedimientos judiciales que hacen parte de ésta.
En lo atinente a la conformación misma de la Comisión, si bien se mantiene una estructura muy similar en cuanto a las personas convocadas a formar parte de ella, hay una diferencia importante ya que se deja abierta la posibilidad de que por iniciativa propia se convoque a otros participantes.

En efecto, el gobierno ha estimado que la Comisión debe estar presidida por el Ministro del Interior y de Justicia y conformada por el Secretario jurídico de la Presidencia de la República, el Ministro de Hacienda y Crédito Público, los Magistrados de la Sala de Consulta y Servicio Civil del Consejo de Estado, un Magistrado por cada una de las Secciones del Consejo de Estado; pero además se establece expresamente que la Comisión tiene la facultad de invitar a los funcionarios, representantes de las entidades, expertos, académicos y demás personas que estime puedan ser de utilidad para los fines de la Comisión cuando así lo estime necesario, con la finalidad de cumplir los objetivos encomendados.

Como se ve, si bien se hace un intento por hacer de éste un ejercicio incluyente es de carácter meramente facultativo, pues no solo la invitación a participantes diferentes es opcional; sino que además las sugerencias presentadas por esos otros actores no tienen carácter vinculante, puesto que el Decreto no contempla en ninguno de sus apartes un verdadero mecanismo de participación, entendida ésta como la opción cierta de incidir directamente en el contenido mismo del proyecto de ley.

En este punto, se hace necesario observar la forma en la cual se concibió el rol de la Comisión y sobretodo la forma en la que, al menos desde lo teórico ésta debió cumplir su tarea.

\section{LA LABOR CUMPLIDA POR LA COMISIÓN DE REFORMA AL CÓDIGO CONTENCIOSO ADMINISTRATIVO COLOMBIANO}

El Decreto define cuatro etapas en las que se debe surtir el proceso de creación normativa realizado 
por la Comisión. En primer lugar, se debían definir los grandes temas objeto de reforma, para lo cual, se instó al desarrollo de actividades encaminadas a establecer la orientación general que se quería para el código. Una vez agotada esta primera etapa debería pasarse, a lo que el gobierno nacional denominó, a la definición de los detalles de cada una de las partes integrantes del código al cabo de lo cual, y como parte de una tercera etapa, debía procederse a la redacción y corrección del proyecto.

Finalmente, se contempló la socialización del proyecto antes de que se efectuara su presentación en el Congreso. Dicha socialización debía ser realizada a través de la realización de foros y seminarios en los que debían participar los Magistrados y Jueces, Congresistas, abogados, académicos, ex Magistrados, funcionarios de entidades estatales y ciudadanos en general.

De este modo, se le dieron amplias facultades a la Comisión para que realizara su trabajo; pero, dado que el objetivo es determinar si en la labor realizada se observa un proceso de construcción normativa reflexivo se debe establecer la presencia o la ausencia de los siguientes elementos: una actitud de permanente aprendizaje, la capacidad de autocuestionamiento y autocorrección, la apertura hacia mecanismos creativos, flexibles y/o extrajurídicos y la asociación de los destinatarios de las normas al proceso.

Una vez iniciadas las labores, la Comisión se reunió en varias oportunidades con los miembros de la rama jueces y magistrados con quienes se estudió la problemática interna, las preocupaciones y sugerencias alrededor de la reforma; esto generó espacios de participación activa en los que se partió de una mirada crítica no solamente al texto anterior, sino a la forma en la cual se venían implementando las normas llamadas a ser reformadas. Ello implicó un proceso de auto evaluación seguido por la formulación de propuestas que estaban llamadas a formar parte del proyecto de ley. En ese proceso se acudió a diversos estudios en dos frentes bien diferenciados: el antes y el después de la reforma.
Así, se evaluaron entre otras, las estadísticas de congestión judicial en la totalidad del territorio nacional, lo cual permitió ver las distintas problemáticas en cada región del país, los índices de percepción de la ciudadanía frente a la actividad ejercida por los funcionarios de la jurisdicción y, la proyección sobre el impacto que para los proceso en curso y para las garantías de los ciudadanos tendría por ejemplo, la adopción de la oralidad por ser éste uno de los temas centrales de la reforma.

En segundo orden de importancia, se encuentra la intervención de académicos de las más importantes universidades del país, quienes a través de ponencias en numerosos seminarios tuvieron la oportunidad de realizar observaciones y sugerencias sin que hubiese un mecanismo diferente a la voluntad de los miembros de la Comisión que permitiera consolidar dichas observaciones en algo más que en sugerencias de buena fe.

Además de lo anterior, como parte de las labores previas a la formulación del proyecto, se previó convocar a una comisión de cooperación técnica conformada por reconocidas personalidades del mundo del derecho público francés; dentro de la que se destaca la presencia de Consejeros de Estado y reputados profesores universitarios, quienes desde fuera, hicieron algunas observaciones frente a la forma en la que se venía ejerciendo la actividad jurisdiccional, las falencias de la codificación anterior y las nuevas oportunidades que se presentan para darles solución a través de la reforma.

Cabe resaltar los esfuerzos realizados por la sala de consulta y de servicio civil del Consejo de Estado colombiano, cuyos Magistrados forman parte de la Comisión de Reforma; no sólo en materia de realización de eventos de intercambio de ideas alrededor del contenido del proyecto de ley, sino también sobre la función consultiva y la calidad de la legislación.

En ese orden de ideas, se realizó un evento conjunto con las Embajadas de España y de Francia 
en Colombia en el que se debatieron temas relacionados con la necesidad de desarrollar técnicas legislativas basándose en las normas y políticas europeas y españolas en la materia, así como en la experiencia del Consejo de Estado español en la revisión de textos normativos. (CONSEJO: 2009: 31) Dicho evento contó con la participación de jueces y magistrados colombianos, franceses y españoles, representantes de los poderes legislativo, ejecutivo y judicial, así como las universidades y otros sectores académicos.

Estas son claras muestras tanto de la apertura hacia un aprendizaje continuo como hacia la autoevaluación y la autocorrección, necesarias para un proceso de construcción normativa reflexivo. En cuanto a la apertura hacia mecanismos creativos, flexibles y/o extrajurídicos puede verificarse, por ejemplo, la utilización de las modernas tecnologías de información y comunicación tanto en el proceso de redacción del código como en su contenido; puesto que, una de las novedades más importantes en materia procedimental está relacionada con la inclusión en el capítulo cuarto del proyecto de la utilización de medios electrónicos en el procedimiento administrativo, partiendo siempre de la base de la voluntariedad.

Finalmente, en lo relativo a la asociación de los destinatarios de las normas al proceso, es oportuno mencionar que en Colombia, con la entrada en vigencia del texto constitucional de 1991 se pretendió pasar de la democracia representativa a la democracia participativa; por lo cual, el principio de participación ciudadana se consagró como un valor constitucional y como un derecho fundamental de los ciudadanos.

Dicha consagración impone, como es natural obligaciones en cabeza del Estado con miras a contribuir a la organización, promoción y capacitación de las asociaciones cívicas, sindicales, comunitarias, juveniles, benéficas o de utilidad común no gubernamentales; con el objeto de que constituyan mecanismos democráticos de representación en las diferentes instancias de participación, concertación, control y vigilancia de la gestión pública que se establezcan.
En la labor ejercida por la comisión de reforma al código, este objetivo facilitador de la participación ciudadana fue limitado e insuficiente; por cuanto el único mecanismo previsto para escuchar al ciudadano del común fue la inclusión de un link en la página Web del Consejo de Estado en el que se podían consultar libremente las memorias de los seminarios de discusión realizados y presentar los comentarios o sugerencias pertinentes.

En este aspecto, se destaca la utilización de herramientas electrónicas novedosas que facilitaron el ingreso y procesamiento de las iniciativas ciudadanas, pero se llama la atención frente a la enorme distancia que separa, aún hoy en un país como Colombia, al ciudadano del común del acceso a la tecnología; por lo que este mecanismo si bien podría ser eficiente en un escenario distinto, en las condiciones actuales es más bien excluyente.

Esa es la razón por la cual, no debe sorprendernos que el índice de participación a través de ese mecanismo haya sido tan limitado y si a la brecha tecnológica se le suma el analfabetismo electrónico y la ausencia de mecanismos que aseguren que por lo menos las iniciativas ciudadanas van a ser objeto de discusión por parte de la Comisión redactora, es posible aventurarnos a concluir que en el proyecto presentando al Congreso está ausente la visión del destinatario final de los procedimientos administrativos.

Es por ello, que puede afirmarse que en el panorama actual colombiano, la garantía al derecho fundamental de participación ciudadana se da fuera del ámbito de creación normativa limitándose los espacios de participación en ese aspecto a la iniciativa popular legislativa y al control ex post de los textos, ya convertidos en leyes que se efectúa a través del ejercicio de la acción pública de inconstitucionalidad; esto dista aún mucho del principio de democratización de la democracia, propio de los procesos de construcción normativa reflexivos. 


\section{CONCLUSIONES}

De acuerdo con lo propuesto en la parte introductoria de este escrito, al cotejar las características que definen los procesos de creación normativa modernos con una de las más recientes experiencias en relación con el ejercicio de esa actividad en Colombia, esto es, el proceso de construcción del proyecto de ley de reforma al código contencioso administrativo, pudo establecerse que pese a que en la dinámica contemporánea los ciudadanos están llamados a participar de manera directa en ellos, dejando atrás la idea de participación exclusivamente a través de la representación, aún persiste un alto índice de inactividad ciudadana en el escenario normativo.

Dicha inactividad e incluso apatía puede ser explicada de distintas maneras. En primer lugar, se encuentra el analfabetismo político contra el cual poco se ha hecho a nivel estatal, que se concreta en un total desconocimiento de los espacios de interacción entre las distintas autoridades estatales y los ciudadanos y, de la obligatoriedad de dichas autoridades para promoverlos y asegurarlos.

En segundo lugar, debe mencionarse la desconfianza en la efectividad real de dichos espacios, pues en los contados casos en los cuales los ciudadanos tienen conocimiento de algunos de los mecanismos de participación ciudadana, dicho conocimiento va generalmente acompañado de un arraigado sentimiento de incredulidad frente al carácter vinculante y la seriedad con la que son tomadas las iniciativas ciudadanas por parte las autoridades públicas.

En tercer lugar, se encuentra una dificultad de carácter generacional originada en el hecho de que pese a que el texto constitucional está próximo a cumplir veinte (20) años de vigencia, un número importante de ciudadanos políticamente activos desarrolló su actividad en vigencia de la constitución de 1986 y no han logrado desarraigar los viejos conceptos y formas de participación; de esta manera, estaríamos en presencia de un período de transición cuya duración dependerá en gran medida de la adopción de medidas educativas y de apropiación del quehacer público por parte de los ciudadanos.

Sin embargo, la labor educativa no debe estar enfocada únicamente a los ciudadanos, sino que debe ser en doble vía puesto que las mismas autoridades estatales en muchos casos desconocen la importancia que para el adecuado ejercicio de sus funciones tiene concretar el mandato constitucional de promoción de la participación ciudadana.

El hecho de que para los constructores del derecho contemporáneo la ausencia de participación ciudadana no constituya un factor inhabilitante para el ejercicio de sus funciones necesariamente repercute en la calidad de las leyes que crean, pues tal como se expuso a lo largo del artículo, ésta depende de que sea producto de una mayor reflexión. A su vez, el nivel de reflexión de un proceso de creación normativa está directamente relacionado con factores tales como la pluralidad, la asociación de los destinatarios de las normas, la practicidad, la adaptabilidad, la flexibilidad y la apertura hacia un aprendizaje permanente.

En nuestro caso, al no ser vinculantes las iniciativas ciudadanas, no puede hablarse de una verdadera negociación entre gobernantes y gobernados por lo que las normas terminan siendo la expresión de un proceso unilateral altamente influenciado por grupos de presión que va en contravía de lo que sería deseable y, que en últimas termina adoptando el rostro de ineficiencia administrativa.

Tampoco puede afirmarse que, los nuestros, sean procesos gerenciales por cuanto aunque se ha avanzado, por ejemplo a nivel de integración de las nuevas tecnologías de información y de comunicación no se evidencia ningún tipo de actividad de autoevaluación por parte de los hacedores del derecho; por consiguiente, su capacidad de renovación y de creatividad en los procesos está ausente y evidencia la 
necesidad de promover una modificación en su comportamiento que privilegie la reflexión y la calidad, sobre la inmediatez y la necesidad de mostrar productos.

Vale la pena resaltar cómo aunque la preocupación por tecnificar la producción normativa lleva en el contexto internacional un recorrido de más de 30 años, entre nosotros es un tema muy reciente al que apenas hace muy poco empezaron a referirse las autoridades estatales, se ha concretizado, por el momento, en la ley de modernización del Congreso de la República y en la adopción de directrices de técnica normativa vía decreto, que si bien constituyen un importante esfuerzo, no son suficientes para asegurar una mejor calidad de las leyes.

En consecuencia, entre nosotros aún no se ha planteado la discusión alrededor de la posibilidad de que se comprometa la responsabilidad de los creadores del derecho cuando quiera que los resultados de su actividad no sean satisfactorios, por lo que permanecemos a merced de su buena fe.

En suma, si bien se ha avanzado sobretodo en el interés en de las autoridades públicas por conocer e implementar las modernas técnicas de producción normativa con miras a hacer de las normas vigentes normas realmente eficaces, aún hay mucho por hacer especialmente en lo relacionado con lograr que el derecho sea el resultado de un diálogo social equitativo, y no de imposiciones legitimadas en la representatividad o en la consagración de mecanismos de participación concebidos para no ser usados.

\section{BIBLIOGRAFÍA}

BECK, U., \& WILLMS, J. (2004). Conversations with Ulrich Beck. Citado por Pieret, J. en "D'une Societé du Risque vers un Droit Réfléxif.

http://dev.ulb.ac.be/droitpublic/fileadmin/ telecharger/theme_1/contributions/De_la societe_du_risque_vers_un_droit_reflexif_.p $\overline{\mathrm{df}}$
BELLEY, J.G. Une justice de la seconde modernité: proposition de príncipes généraux pour le prochain code de procédure civile", Revue de droit de Mc Gill, vol. 46, p. 317. Citado por PIRET, Julien. D'une Societé du Risque Vers un Droit Réflex? http://dev.ulb.ac.be/droitpublic/fileadmin/ telecharger/theme_1/contributions/De_la_societe_ du_risque_vers_un_droit_reflexif_.pdf

BOURDIEU, P.\& TEUBNER, G. (2000). LaFuerza del Derecho. Bogotá, Colombia: Universidad de los Andes. Instituto Pensar. Universidad Javeriana. Siglo del Hombre Editores.

CATALANO, P. (1974). Populus Romanus Quirites. G. Giappichelli. Editorial Torino.

CHEVALLIER, J. (2002) L'État Postmoderne, L.G.D.J. Paris, Francia.

CHEVALLIER, J. (2011) L'État Postmoderne, L.G.D.J. (Traducción Libre) Paris, Francia.

CHEVALLIER, T. Paris, Francia.

CONSEIL D'ÉTAT. (2003). Rapport Public du Conseil d'État Considérations Générales: Perspectives pour la Fonction Publique. EDCE. (54).

CONSEJO DE ESTADO. (2009). Sala de Consulta y Servicio Civil. Memoria.

CONSEJO DE ESTADO. (2008). Misión de Cooperación Técnica en Colombia del Consejo de Estado Francés. Seminario Franco Colombiano sobre la Reforma a la Jurisdicción Contencioso Administrativa. Memorias

DUBÈ, R. (2007). Èlèments de thèorie sur les commissions de rèforme du droit et l'innovation cognitive en matière de justice pénale: contributions conceptuelles de Michel Focault et de Niklas Luhmann. Champ pènal/Penal field, nouvelle revue internationale de criminologie [En lìnea], Sèminaire Innovations Pénales, Le systéme de droit. Consultado el 17 de marzo 2010. http://champpenal.revues.org/694

DUBÈ, R. (2007). GARCÍA AMADO, J. A. (1992). Sobre los Modos de Conocer el Derecho o de Cómo Construir el Objeto Jurídico. 
IBAÑEZ NAJAR, J. E. (2004). 20 Años de Vigencia del Código Contencioso Administrativo Colombiano.

ELIAS, N. (1975). La dynamique de l'Occident, 1969, Calmann-Lèvy. Citado por

HOECKE, V. (2002) Law as communication, Oxford, Hart Publishing, 2002. Citado por Pieret, J. En D'une Societé du Risque vers un Droit Réfléxif. http://dev.ulb.ac.be/droitpublic/fileadmin/ telecharger/theme_1/contributions/De_la_societe_ du_risque_vers_un_droit_reflexif_.pdf

JOCHUM, M., \& LEDERMAN, S. (2009). La démarche légistique entre théorie et pratique. www. bk.admin.ch/themen/lang/00938/02124/04794/ index.html

KONNTZ, H., \& WEIHRICH, H. (1998) Administración. McGraw-Hill.

MAESSCHALCK, M. (2008). La Gouvernance Réflexive dans l'Union Européenne. Ponencia presentada durante el I Ciclo de Conferencias "Gouverner par les Normes", organizado por el Centro Parelmen de Filosofía del Derecho de la Universidad Libre de Bélgica febrero-Marzo.
MARTÍN-RETORTILLO BAQUER, L. (2003). La Calidad de la Ley Según la Jurisprudencia del Tribunal Europeo de Derechos Humanos (especial referencia a los casos "Valenzuela Contreras » y «Prado Bugallo » ambos contra España. Revista Derecho privado y Constitución. (17).

PIRET, J. D'une Societé du Risque Vers un Droit Réflexif? http://dev.ulb.ac.be/droitpublic/fileadmin/ telecharger/theme_1/contributions/De_la_societe_ du_risque_vers_un_droit_reflexif_.pdf

PIRET, J. (2003) D'une Societé du Risque Vers un Droit Réflex?, (Traducción libre). Université Libre de Bruxelles, Centre de Droit Public. http://dev.ulb.ac.be/droitpublic/fileadmin/ telecharger/theme_1/contributions/De_la societe_du_risque_vers_un_droit_reflexif__.pdf

WALTON, M. (1992). Cómo Administrar con el Método Deming, Bogotá, Colombia: Norma.

ZÁRATE PÉREZ, A. (2004). La incorporación constitucional de los principios de eficacia y eficiencia administrativa. Bogotá, Colombia: Universidad Externado de Colombia, Colección Tesis de Grado. 